\title{
Estimating Primaries by Sparse Inversion with Cost-Effective Computation
}

\author{
Xiaopeng Zhou ${ }^{1,2}$, Yike $\mathrm{Liu}^{1, *}$ and Lanshu Bai ${ }^{3}$ \\ ${ }^{1}$ Key Laboratory of Petroleum Resource Research, Institute of Geology and \\ Geophysics, Chinese Academy of Sciences, Beijing 100029, China. \\ 2 University of Chinese Academy of Sciences, Beijing 100049, China. \\ ${ }^{3}$ China Earthquake Networks Center, Beijing 100045, China.
}

Received 15 March 2018; Accepted (in revised version) 13 October 2018

\begin{abstract}
Recently, attenuation of surface-related multiples is implemented by a largescale sparsity-promoting inversion where the primaries are iteratively estimated without a subtraction process, which is called estimation of primaries by sparse inversion (EPSI). By inverting for surface-free impulse responses, EPSI simultaneously updates the primaries and multiples, both of which contribute to explaining the input data, and therefore promote the global convergence gradually. However, one of the major concerns of EPSI may lie in its high computational cost. In this paper, based on the same gradient-descent framework with EPSI, we develop a computationally costeffective primary estimation approach in which a newly defined parameterization of primary-multiple model is adopted and an efficiently defined analytical step-length is developed. The developed approach can yield a better primary estimation at less computational cost as compared to EPSI, which is verified by two synthetic datasets in numerical examples. Moreover, we apply this approach to a shallow-water field dataset and achieve a desirable performance.
\end{abstract}

AMS subject classifications: $65 \mathrm{~K} 10,86-08,86-\mathrm{A} 22$

Key words: Inverse problem, multiple removal, primary estimation, impulse response.

\section{Introduction}

Nowadays, multiple removal is still a fundamental step in the conventional seismic data processing $[30,31,34,35]$, even though seismic imaging using multiples is widely investigated in recent years [32,33,36,37]. Multiple removal is implemented as a predictionsubtraction process for many techniques, such as surface-related multiple elimination

*Corresponding author. Email addresses: zhouxiaopeng@mail.iggcas.ac.cn (X. Zhou), ykliu@mail.iggcas.ac.cn (Y. Liu), bailanshu@seis.ac.cn (L. Bai) 
(SRME) [4, 26], which has achieved a widespread adoption in industry due to its practicality. Despite its major success over the years, SRME still have limitations in many cases, such as: 1) subtraction based on minim energy constraint can hardly yield a satisfactory result when primaries and multiples interfere with each other $[12,15] ; 2)$ as the strongest contributor of multiples, the near-offsets of the input data are absent due to the acquisition gap especially for shallow water data, which makes it a necessity for nearoffset data reconstruction $[2,7,27]$. Therefore, geophysicists increasingly shift their attentions towards the demultiple methods that the primary estimation is implemented with an explicit full-wave inversion [10,11, 14, 19, 20, 25, 28, 29]. Among these methods, the approach of estimation of primaries by sparse inversion (EPSI) attracts great attention. This approach integrates nears-offset data reconstruction into multiple removal without a subtraction process [19].

By parameterizing primaries with wavelets and spike-like impulse responses, EPSI directly estimates the primaries through inversion, which potentially conserves the primaries even in case that primaries and multiples seriously interfere with each other [10]. Additionally, with its new insight into geophysical signal separation, EPSI has been successfully applied in various aspects, such as passive seismic data, blended data and ocean-bottom cable data [1,9, 18,21-24]. Despite its great success in many cases [17], the EPSI algorithm still has some limitations.

As the impulse response is parameterized as a series of spikes, lots of free parameters need to be defined to determine the sparsity of the gradient in each iteration. This shortcoming limits the flexibility and practicality of the EPSI algorithm, and may ultimately affect the reliability of the final result [10]. Therefore, some geophysicists made refinements for the EPSI algorithm to eliminate the number of free parameters $[10,11,14]$.

Another challenge faced by EPSI may lies in its high computational cost. To ensure the convergence and minimize the artifacts, a large number of iterations are required in general. Specifically, in each iteration, the spike-like impulse response is used to update the primaries and multiples, both of which explain the input data until the energy of residual is controlled in an acceptable scope. Usually, tens of iterations, with each one involving a multi-dimensional correlation and convolution, are needed for a satisfactory final primary estimation. Therefore, reducing the computational cost or accelerating the convergence is an important issue for EPSI $[6,8,13]$. In addition, the spike-like impulse response is determined through imposing the sparsity to the gradient by the practitioner (due to the sparsity and causality). When the multiples in the input data are explained with a low rate, the multiple impulse response would be wrongly selected as the primary impulse response, which cannot be eliminated in the subsequent iterations and therefore causes an irretrievable result. Thus, accelerating the convergence not only results in a lower computational cost, but potentially contributes to a more reliable primary estimation.

Based on the same gradient-descent framework with the EPSI algorithm, we develop a computationally efficient algorithm in which a newly defined parameterization of primary-multiple model is adopted for accelerating the convergence, and an analyti- 
cal step-length is concisely derived in each update for reducing the computation further. It should be noted beforehand that, in this paper, the scope of our developed approach is limited in primary estimation, while near-offset data reconstruction is not involved. The developed approach can potentially yield a better primary estimation when taking a less computation time as compared to the EPSI algorithm, which is illustrated by two synthetic datasets and a shallow-water field dataset. In what follows, we first review the algorithm of EPSI in the Section 2.1. Then we describe the algorithm of our developed approach in the Section 2.2. Last, we demonstrate the effectiveness and practicality of our developed approach with three numerical examples when compared with EPSI.

\section{Methodology}

\subsection{Estimation of primaries by sparse inversion (EPSI)}

According to the detail-hiding operator notation introduced by [3], the upgoing data $\mathbf{P}$ recorded at the surface can be expressed as

$$
\mathbf{P}=\mathbf{X}_{0} \mathbf{S}+\mathbf{X}_{0} \mathbf{R} \mathbf{P}
$$

where $\boldsymbol{X}_{0}$ represents the primary (including internal multiple) impulse response of the subsurface, $\mathbf{S}$ and $\mathbf{R}$ represent the source wavelet and surface reflectivity respectively. Assuming $\mathbf{S}(w)=S(w) \mathbf{I}$ (a constant-source wavelet for all shots), and $\mathbf{R}=-\mathbf{I}$ (the reflectivity is -1) in Eq. (2.1), EPSI directly estimates the unknowns $\left(\boldsymbol{X}_{0}\right.$ and $\left.S\right)$ through a full-wave inversion process such that primaries $\mathbf{X}_{0} \mathbf{S}$ and multiples $\mathbf{X}_{0} \mathbf{R} \mathbf{P}$ can be simultaneously obtained [19]. EPSI is implemented by minimizing the following objective function:

$$
J=\sum_{w} \sum_{j, k}\left|\mathbf{V}_{i}\right|_{j, k}^{2}
$$

where $\mathbf{V}$ is the residual, which is defined as $\mathbf{V}=\mathbf{P}-\mathbf{X}_{0} S+\mathbf{X}_{0} \mathbf{P} ; i$ is the iteration number; $j$ and $k$ represent the shot and receiver index of $\mathbf{V}_{i}$ respectively; $w$ is the frequency. During the inversion, $\mathbf{X}_{0}$ is assumed to be a series of spikes in time domain. Based on the steepestdescent method, the updates $\Delta \mathbf{X}_{0}$ is introduced as

$$
\Delta \mathbf{X}_{0}=\mathbf{V}_{i}\left(S_{i} \mathbf{I}-\mathbf{P}\right)^{H},
$$

where $(\bullet)^{H}$ represents the conjugate transpose operation. Before adding $\Delta \mathbf{X}_{0}$ to the current primary impulse response, the sparsity is imposed to $\Delta \mathbf{X}_{0}$ by the practitioner. Specifically, in each iteration, a space-time window is placed over $\Delta \mathbf{X}_{0}$; and in this window, the sample with the maximum amplitude per trace is selected as the spike [20]. With the iteration continuing, the window is gradually expanded both in space and time direction such that all the primary events can be selected. As a result, $\mathbf{X}_{0}$ can be iteratively updated:

$$
\mathbf{X}_{0, i}=\mathbf{X}_{0, i-1}+\alpha \Delta \mathbf{X}_{0}
$$


The step-length $\alpha$ can be obtained with a line-searching method [19]. Because of the constant source wavelet assumption, $S_{i}$ can be updated in the same way as $\mathbf{X}_{0}$, or estimated by matching $\mathbf{X}_{0, i}$ with $\mathbf{P}+\mathbf{X}_{0, i} \mathbf{P}$ in time domain [19]. Consequently, primaries and multiples can be simultaneously obtained:

$$
\left\{\begin{array}{l}
\mathbf{M}_{0, i}=\mathbf{X}_{0, i} \mathbf{P}, \\
\mathbf{P}_{0, i}=\mathbf{X}_{0, i} S_{i}, \quad \text { or } \quad \mathbf{P}_{0, i}=\mathbf{P}-\mathbf{M}_{0, i} .
\end{array}\right.
$$

The essence of EPSI is to find a correct impulse response model that can simultaneously explain all the primaries and multiples in input data, such that the primaries can be directly estimated without a subtraction process. However, the amplitude of the inverted primary impulse response is highly associated with the relative strength between primaries and their related multiples. Therefore, the success of EPSI highly depends on the internal consistency of the relative amplitude between primaries and multiples in the input data [10]. However, it is hard to accurately explain all the input data with the assumptions of a constant source-wavelet and constant surface reflectivity used in the primary-multiple model (Eq. (2.1)) due to some inaccuracies in the primary-multiple model, such as ghost, phase variation, 3D wavefield effect and directivity of sources and receivers in the measured data.

Thus, some multiple residual can be hardly explained, which leads to a low convergence rate, especially at intermediate or late iterations. Consequently, when imposing the sparsity to the gradient, the impulse response of the unexplained multiples (or multiple residual) may be easily selected as the primary impulse response, which cannot be eliminated in the subsequent iterations and therefore causes an irretrievably wrong result. Here, we use a 1D synthetic data example to clearly illustrate the issue.

An original shot-gather (Fig. 1a) where only the first event is the primary, is used as the input for the EPSI algorithm. During imposing the sparsity to the gradient in the first 30 iterations, the time-length of the picked window is controlled so that only the primary impulse responses are selected and their related multiples can be eliminated as much as possible. However, although the primary impulse response is successfully inverted after 30th iterations (Fig. 1b), some multiples still remains in the residual (unexplained data), as indicated by arrows in Fig. 1c. In this case, if the picked window is expanded in time direction with the iteration continuing (for most general situations), the multiple impulse response would be probably selected as the primary impulse response inevitably. This is demonstrated in the inverted primary impulse response at the 40th iteration (Fig. 1d), where the arrows indicate the wrongly picked impulse responses. As a result, some fake primary events will be generated in the primary estimation, and these primary events cannot be eliminated in the subsequent iterations.

\subsection{Estimating primaries by sparse inversion with cost-effective computation}

\subsubsection{Description of our developed approach}



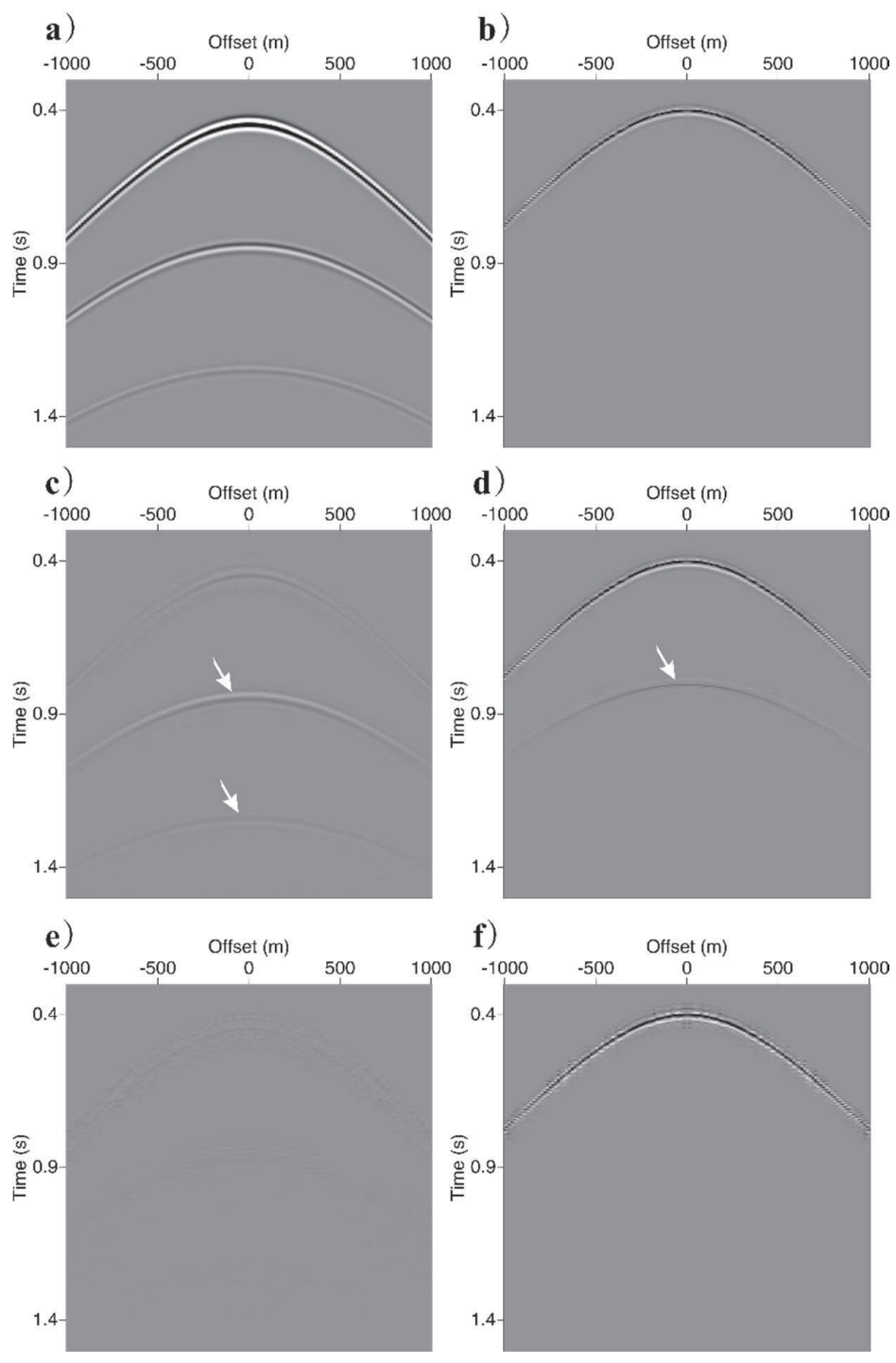

Figure 1: (a) The original shot-gather generated from a 1D model with a single reflector; (b) primary impulse response inverted by EPSI after 30 iterations; (c) the residual of EPSI after 30 iterations; (d) primary impulse response inverted by EPSI after 40 iterations; (e) the residual of our developed approach after 15 iterations; (f) the primary impulse response of our developed approach after 15 iterations.

To address the aforementioned issue faced by EPSI, we develop a primary estimation approach which adopts a new parameterization of primary-multiple model. The developed approach potentially explains the input data with high efficiency, which provides an alternative for EPSI in many cases.

We begin with an illustration for primary impulse response (shown in Fig. 2) to introduce the new parameterization of primary-multiple model used in our developed ap- 


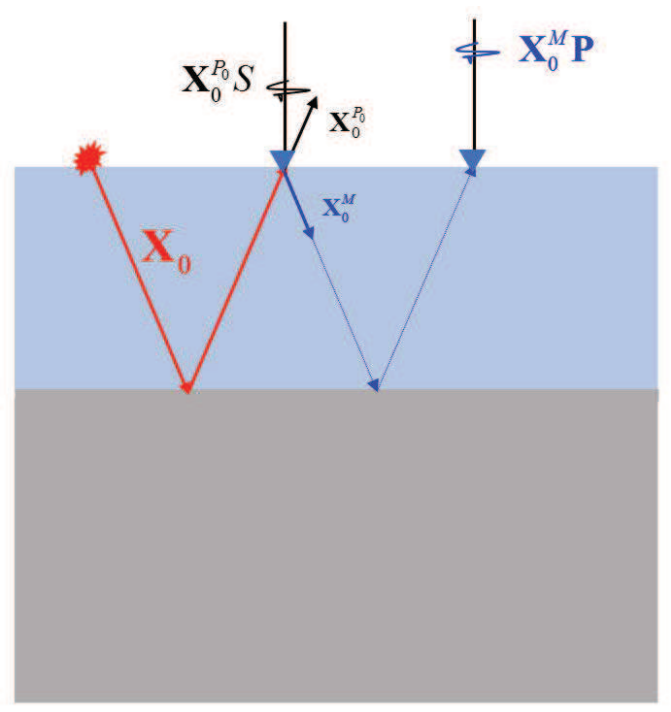

Figure 2: An illustration of impulse responses that contribute to primaries $\left(\mathbf{X}_{0}^{P_{0}}\right)$ and multiples $\left(\mathbf{X}_{0}^{M}\right)$.

proach. As shown in Fig. 2, the impulse response $\mathbf{X}_{0}$ can be viewed as primary reflections of the subsurface excited with an impulsive source wavelet. After reflecting from the subsurface, one part of energy of $\mathbf{X}_{0}$ transmits away from the surface (or directly received by the geophones), which forms the primaries; the other part of energy of $\mathbf{X}_{0}$ reflects against the surface and ultimately forms the multiples. Therefore, the total primary impulse response $\mathbf{X}_{0}$ can be written as a summation of two parts:

$$
\mathbf{X}_{0}=\mathbf{X}_{0}^{P_{0}}+\mathbf{X}_{0}^{M}
$$

where $\mathbf{X}_{0}^{P_{0}}$ and $\mathbf{X}_{0}^{M}$ respectively represent the primary impulse response that only contributes to primaries and only contributes to multiples, and these two terms are mutually independent. Actually, the expression in Eq. (2.6) is also indicated in the EPSI algorithm, where the update of primary impulse response $\Delta \mathbf{X}_{0}$ consists of two parts $\left(\mathbf{V}_{i} S_{i}^{H}\right.$ and $\mathbf{V}_{i} \mathbf{P}^{H}$ ) (see Eq. (2.3)). These two parts respectively represent the primary impulse responses that are transferred from primaries and transferred from multiples in the data residual $[19,20]$.

Thus, based on the Eq. (2.6), the parameterization of the primary-multiple model in Eq. (2.1) can be modified as

$$
\mathbf{P}=\mathbf{X}_{0}^{P_{0}} S+\mathbf{X}_{0}^{M} \mathbf{P} .
$$

Compared with the Eq. (2.1), the directivity of the receiver and the strength of the reflectivity at the surface are incorporated in Eq. (2.7), which loosens the internal consistency of amplitude between primaries and multiples.

As there are three unknowns $\left(\mathbf{X}_{0}^{P_{0}}, \mathbf{X}_{0}^{M}\right.$ and $\left.S\right)$ in Eq. (2.7), we need extra constraints that 1) the impulse response is comprises of a series of spikes and 2) the length of the 
source-wavelet is finite in time domain. The unknowns can be alternately inverted based on the same gradient-descent framework with the EPSI algorithm, so that the multiples $\mathbf{X}_{0}^{M} \mathbf{P}$ and the primaries $\mathbf{X}_{0}^{P_{0}} S$ can be sequentially obtained. The objective function is introduced as

$$
J=\sum_{w}\left\|\mathbf{P}-\mathbf{X}_{0, i}^{P_{0}} S_{i}-\mathbf{X}_{0, i}^{M} \mathbf{P}\right\|^{2}+\lambda L_{s}\left(\mathbf{X}_{0, i^{\prime}}^{P_{0}} \alpha \mathbf{X}_{0, i}^{M}\right)
$$

where $i$ denotes the iteration number; $\sum_{w}$ indicates a summation for all frequencies. $L_{s}$ represents the sparse constraint operator, which is implemented in the same way as EPSI. The constant $\alpha$ ensures $\mathbf{X}_{0, i}^{P_{0}}$ and $\mathbf{X}_{0, i}^{M}$ have the same scale during imposing the sparse constraint.

More details of our developed algorithm are present in Algorithm 1. In this algorithm, all the unknowns are initialized to be zeros in the first iteration (line 2). After that, $\mathbf{X}_{0}^{P_{0}}$ and $\boldsymbol{X}_{0}^{M}$ are alternatively updated based on the gradient-descent method in each iteration (lines 3-6). The gradients of $\mathbf{X}_{0, i}^{P_{0}}$ and $\mathbf{X}_{0, i}^{M}$ are obtained by taking partial derivatives to the first term of the objective function (line 4). However, this cannot yield unique solutions for $\mathbf{X}_{0, i}^{P_{0}}$ and $\mathbf{X}_{0, i}^{M}$. Thus, the sparsity needs to be imposed to the gradients (line 5), as indicated in the second term of the objective function. The way of imposing the sparsity is same with EPSI, with which the event with the maximum amplitude of the gradients is

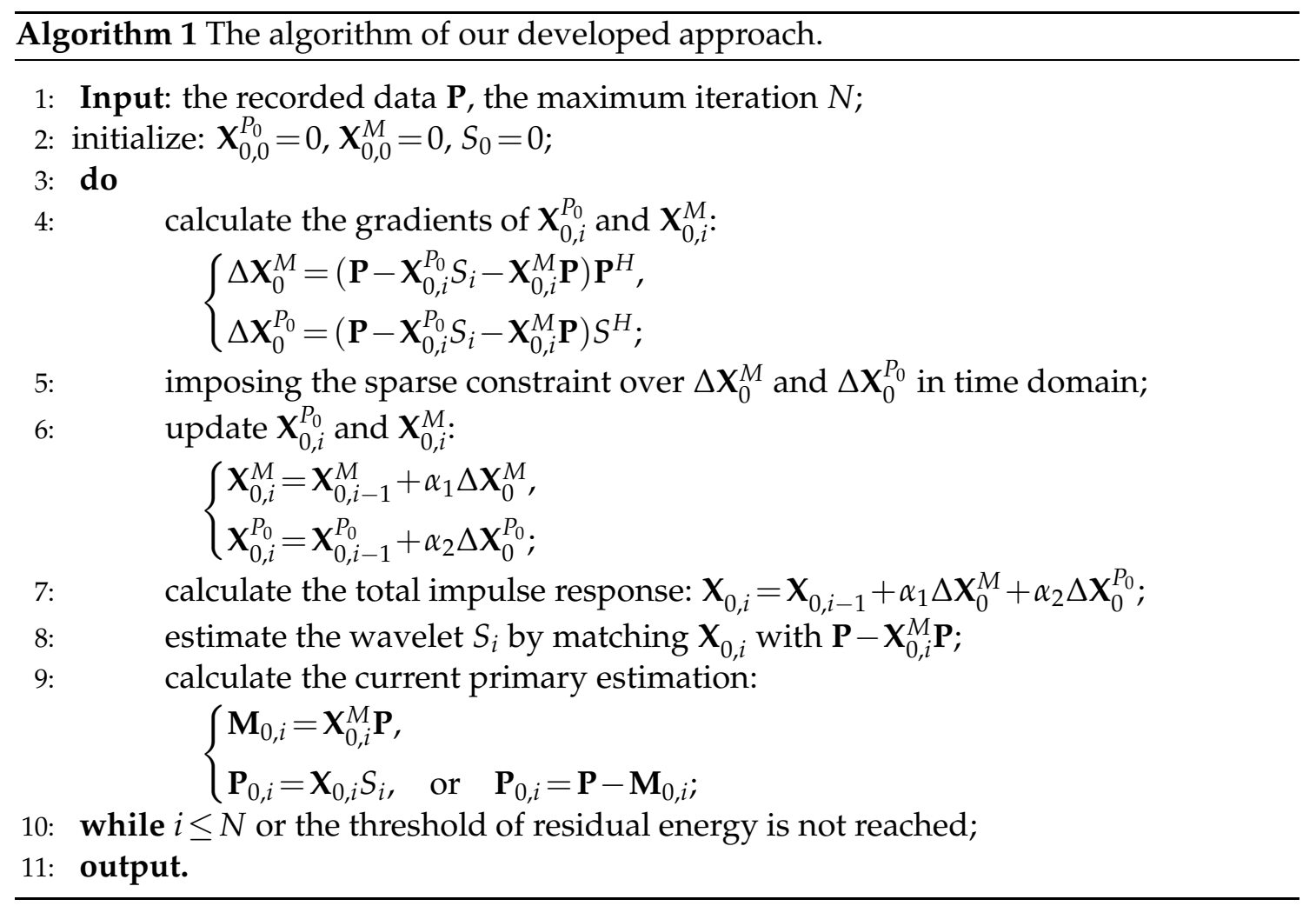


selected in a space-time window. The size of the window is related to iteration number. Generally, to improve the convergence, the window can be kept small in the initial iterations and expanded rapidly with the iteration continuing. Subsequently, $\mathbf{X}_{0}^{P_{0}}$ and $\mathbf{X}_{0}^{M}$ can be updated with their own gradients and a proper step-length (line 6). Instead of using a line-searching method for acquiring the step-length in the EPSI algorithm [19], we concisely derive two analytical step-lengths for updating $\mathbf{X}_{0}^{P_{0}}$ and $\mathbf{X}_{0}^{M}$ in this paper, which is detailedly introduced in Appendix A. Once $\mathbf{X}_{0}^{M}$ is obtained, the multiples $\mathbf{X}_{0, i}^{M} \mathbf{P}$ and the conservative primary estimation $\mathbf{P}-\mathbf{X}_{0, i}^{M} \mathbf{P}$ in the current iteration can be obtained.

The source wavelet $S_{i}$ can also be calculated with the same gradient-descent method, or can be estimated by matching the impulse responses with the conservative primary estimation in the current iteration. In this paper, we choose the latter approach to obtain $S_{i}$. As $\mathbf{X}_{0}^{P_{0}}$ and $\mathbf{X}_{0}^{M}$ contain the same kinetic information, both of them contribute to the direct primary estimation. Therefore, the total impulse response $\mathbf{X}_{0, i}$ is calculated for matching with $\mathbf{P}-\mathbf{X}_{0, i} \mathbf{P}$ to obtain $S_{i}$ (lines 7-8), so that the primaries $\mathbf{X}_{0, i} S$ can be simultaneously obtained (line 9). This contributes to improving the stability of the inversion and protecting primaries, which will be further discussed at the end of Section 2.2.2. Once the residual energy or the iteration number is reached the predefined value, the loop will be terminated (line 10).

\subsubsection{Comparison of EPSI and our developed approach}

In the EPSI algorithm, the multiple is explained with $\Delta \mathbf{X}_{0}$ (see Fig. $3 \mathrm{~b}$ ), which is comprised of two parts (indicated in Eq. (2.3)): the first part $\mathbf{V}_{i} S_{i}^{H}$ maps the primaries in the residual into $\Delta \mathbf{X}_{0}$; the second part $\mathbf{V}_{i} \mathbf{P}^{H}$ is a multi-dimensional correlation, which maps the multiples in the residual to $\Delta \mathbf{X}_{0}$ [19]. However, when the input data does not have a good amplitude consistency between primaries and multiples, the term $\mathbf{V}_{i} S_{i}^{H}$ (transferred from primaries) would not accurately explain multiples in the residual, which affects the convergence rate.

In our developed approach, a newly defined parameterization for primary-multiple model is adopted. Thus, the multiple is explained with the gradient $\Delta \mathbf{X}_{0}^{M}$ that is transferred from the multiples (see Fig. 3a). This is because that the calculation of $\Delta \mathbf{X}_{0}^{M}$ (line 4 of Algorithm 1) is a multi-dimensional correlation, which maps the multiples in the residual to primary location. Therefore, the multiple residual in the current iteration will be mapped to $\mathbf{X}_{0}^{M}$ in the next iteration, which will conversely explain the multiple residual. It is a close-loop process for explaining multiples with high efficiency.

The new parameterization used in our developed approach loosens the demand for the internal consistency of relative amplitude between primaries and multiples in the input data, and therefore weakens the impact of the inaccuracies of primary-multiple model on inversion. Thus, the input data can be resolved with high efficiency. Additionally, when updating the primary impulse response, analytical step-lengths are deduced for updating two types of primary impulse response simultaneously, which avoids at least two extra times calculation of the objective function as compared to the line- 
a)

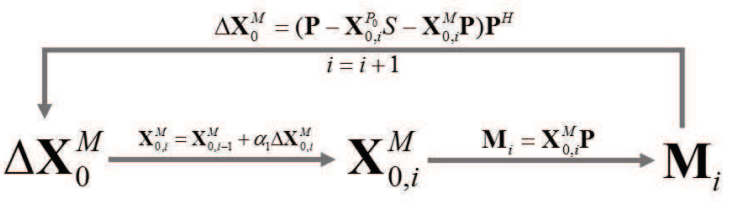

b)

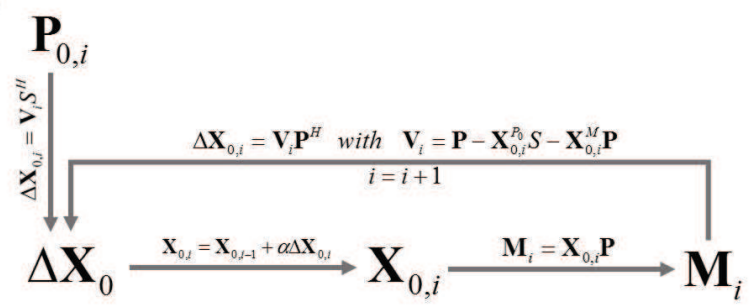

Figure 3: The process for explaining multiples of (a) our developed approach and (b) EPSI.

searching step-length for EPSI in [19], and therefore reduces the computational cost further.

We also use the numerical example presented in Fig. 1 to illustrate the high efficiency of our developed approach. Our developed approach is tested on the original shot-gather (Fig. 1a). When imposing the sparsity to the gradient of impulse responses, we use the same parameters as that for the results in Figs. $1 \mathrm{~b}$ and 1c. The results of our developed approach after 15th iterations are shown in Figs. 1e and 1f, where the data residual (unexplained data) and the primary impulse response are respectively shown. Visually, the residual of our developed approach after 15 iterations (Fig. 1e) contains less multiples as compared to that of EPSI after 30 iterations (Fig. 1c). Therefore, even the picked window is expanded in the time direction with the iteration continuing, the multiple impulse response would not be wrongly selected as the primary impulse response.

However, the new parameterization of our developed approach has its own drawbacks. As the primaries and multiples are parameterized with different impulse responses, the connection of them is weakened. Therefore, our developed approach probably cannot perform well in case that primaries and multiples are strongly interfered with each other. To alleviate this problem, the total primary impulse responses are used to calculate the primaries (lines 7-9 in the Algorithm 1), which can partially address this issue, and therefore ensures that the developed approach can still obtain a satisfactory result for cases where primaries and multiples are slightly interfered. Overall, due to the efficiency, the developed approach provides an alternative for EPSI in many cases.

\section{Numerical examples}

In this section, we use three numerical examples including two synthetic datasets and a shallow-water field dataset to demonstrate our developed approach. In each example, 
the effectiveness of our developed approach is demonstrated by comparing it with EPSI. During inverting for the primary impulse response for the two approaches, the same set of free parameters are used for imposing the sparsity for the gradient of primary impulse response.

\subsection{A three-layer model}

We use a three-layer model, in which the velocity changes only in depth direction, for generating the synthetic dataset. There are 201 shots with $25 \mathrm{~Hz}$ Ricker wavelet sources synthetized in the dataset. All the sources and receivers are overlapped and fixed in the depth of $10 \mathrm{~m}$ below the surface. No deghosting is done for the dataset. One of the shots in the synthetic dataset is present in Fig. 4a, where only the first and the second events are the primaries. Besides, the weak event at around $0.7 \mathrm{~s}$ (indicated by the gray arrow in Fig. 4a) is the internal multiple which also needs to be estimated.

The results of EPSI after 60 iterations are shown in Figs. $4 \mathrm{~b}-4 \mathrm{~d}$, where the primary impulse response, the conservative primary estimation and the residual are sequentially present. Though the primary impulse response are successfully inverted in Fig. 4b, some multiple impulse response (indicated by white arrows) contain in it, which causes some multiples remained in the conservative primary estimation, as indicated by white arrows in Fig. 4c.

The corresponding results of our developed approach after 40 iterations are shown in Figs. 4e-4g. In the total primary impulse response (Fig. 4e) and the conservative primary estimation (Fig. 4f), all the primaries (including internal multiples) are also successfully inverted. Although some multiple residual still exists in our results as indicated by white arrows in Figs. 4e-4f, the residual is largely weakened as compared to that of EPSI (Figs. 4b-4c).

Through the comparison of the two approaches, we conclude that our approach achieves a better primary estimation with less computation cost for this example. This is mainly because that the EPSI algorithm is sensitive to the amplitude consistency between primaries and multiples in the input data. The dataset without deghosting processing would affect the final results for EPSI, because the primary-multiple model in Eq. (2.1) would be no longer accurately satisfied in this case. Whereas for our developed approach, the demand for amplitude consistency between primaries and multiples is loosened as the primaries and multiples are parameterized with different impulse responses (see Eq. (2.7)), and therefore the efficiency can be improved. The comparison of the final residual for the two approaches (Figs. $4 \mathrm{~d}$ and $4 \mathrm{~g}$ ) can also show the difference of the two algorithms. For the residual of EPSI (Fig. 4d), the major primary and multiple events are resolved except some weak imprint. The imprint indicates some low-frequency component of the input data that is not included in the primary-multiple model which is difficult to explain. For the residual of our developed approach (Fig. 4g), the unexplained imprint is evenly distributed, which indicates that our developed approach is not sensitive to the inaccuracies of the input data. 

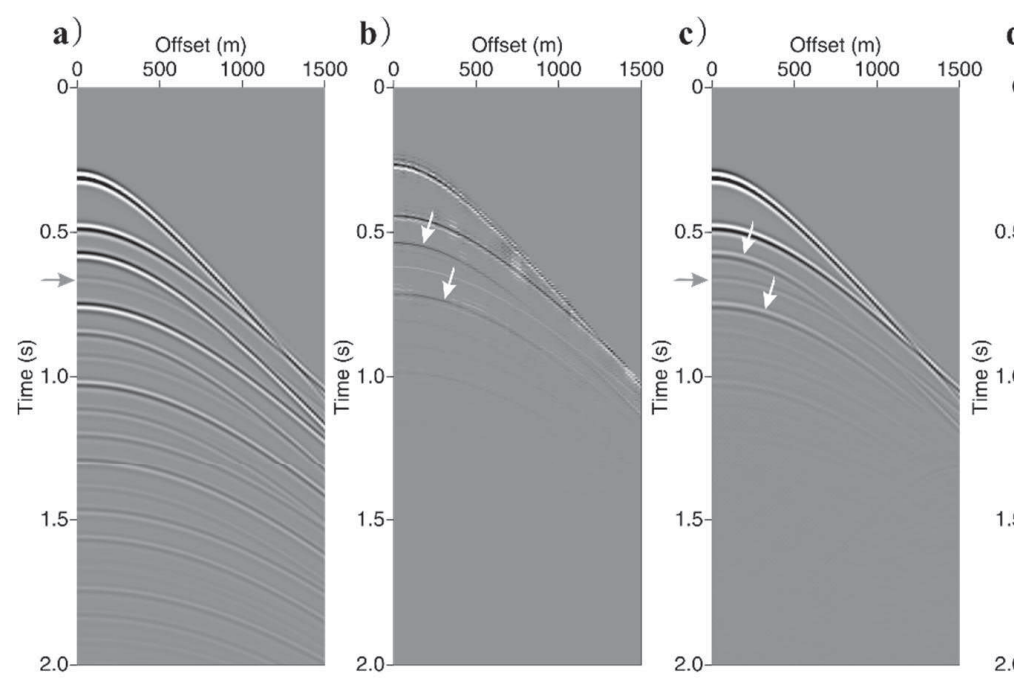

d) Offset (m)
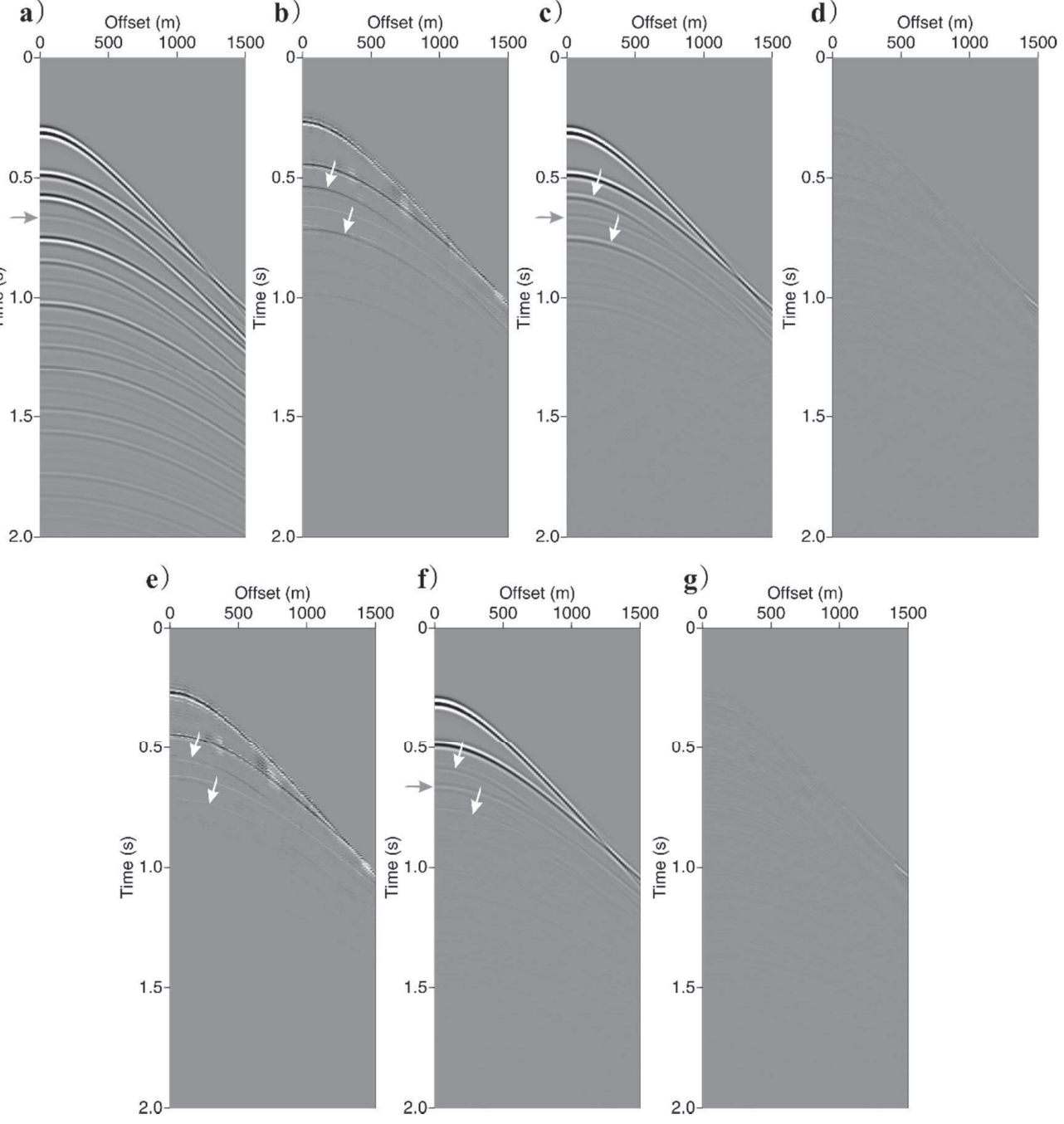

Figure 4: (a) The original shot-gather generated from a three-layer model; (b-d) the primary impulse response, conservative primary estimation and data residual of EPSI after 60 iterations; (e-g) the primary impulse response, conservative primary estimation and data residual of our developed approach after 40 iterations.

\subsection{The Sigsbee 2B model}

For further testing the capability of our developed approach, we use the Sigsbee 2B [16] model as an additional example to generate the synthetic data. The velocity model (Fig. 5) is a part of the original released Sigsbee 2B model. A free surface and strong water bottom (marked by the red dash line) are designed for generating strong surface-related multiples. 181 shots are synthetized with $30 \mathrm{~Hz}$ Ricker wavelet sources, and each shot has 181 receivers with a $10 \mathrm{~m}$ interval. All the sources and receivers are overlapped and placed at $10 \mathrm{~m}$ depth. 


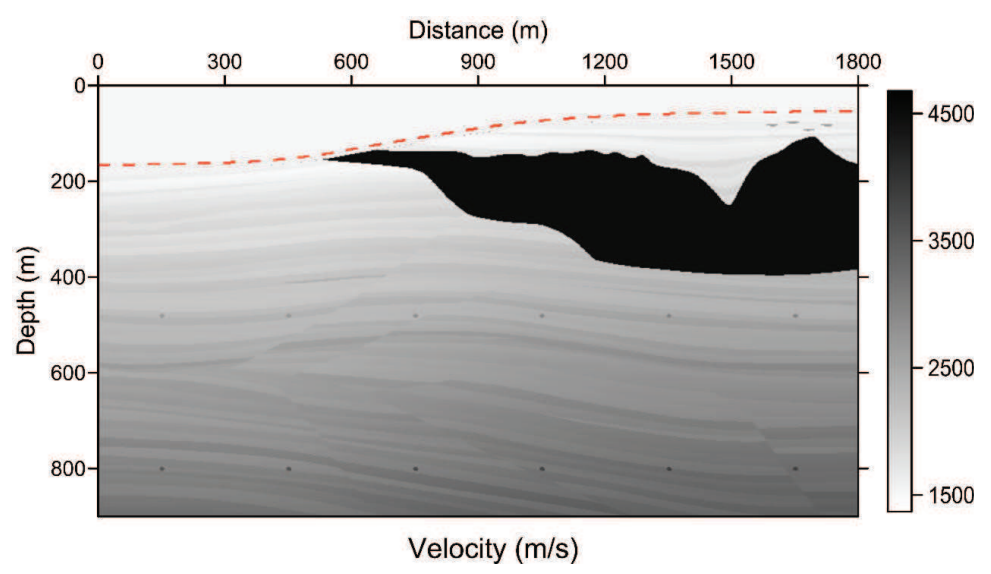

Figure 5: The velocity model used for generating the synthetic data.
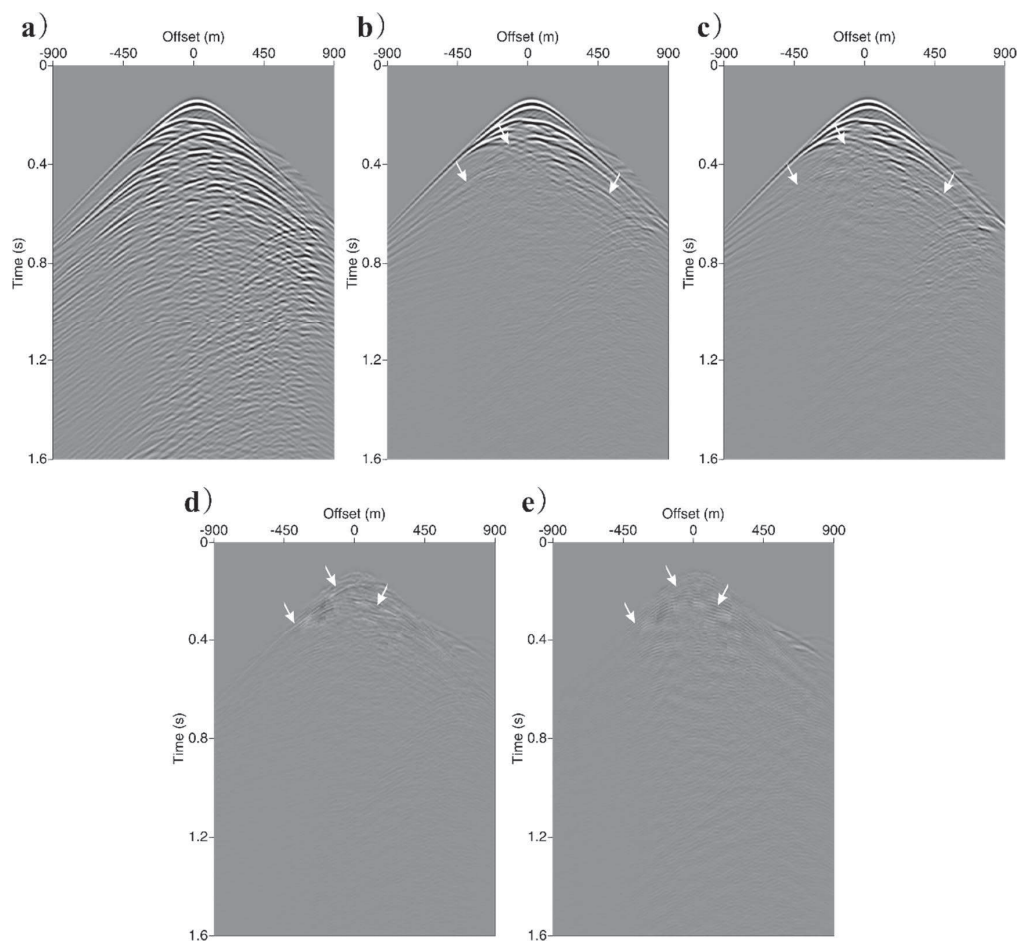

e)

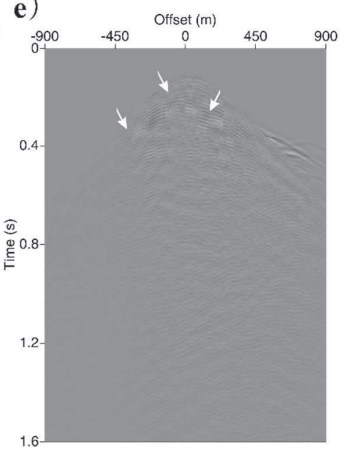

Figure 6: (a) An original shot-gather generated from the velocity model in Fig. 5; (b-c) the conservative primary estimation resulted from EPSI after 60 iterations and our developed approach after 40 iterations; (d-e) the corresponding data residual for results in (b) and (c).

An original shot-gather is shown in Fig. 6a, which is used as the input for EPSI and our developed approach. Figs. $6 \mathrm{~b}$ and $6 \mathrm{c}$ respectively shows the corresponding conservative primary estimation resulted from EPSI after 60 iterations and from our developed 

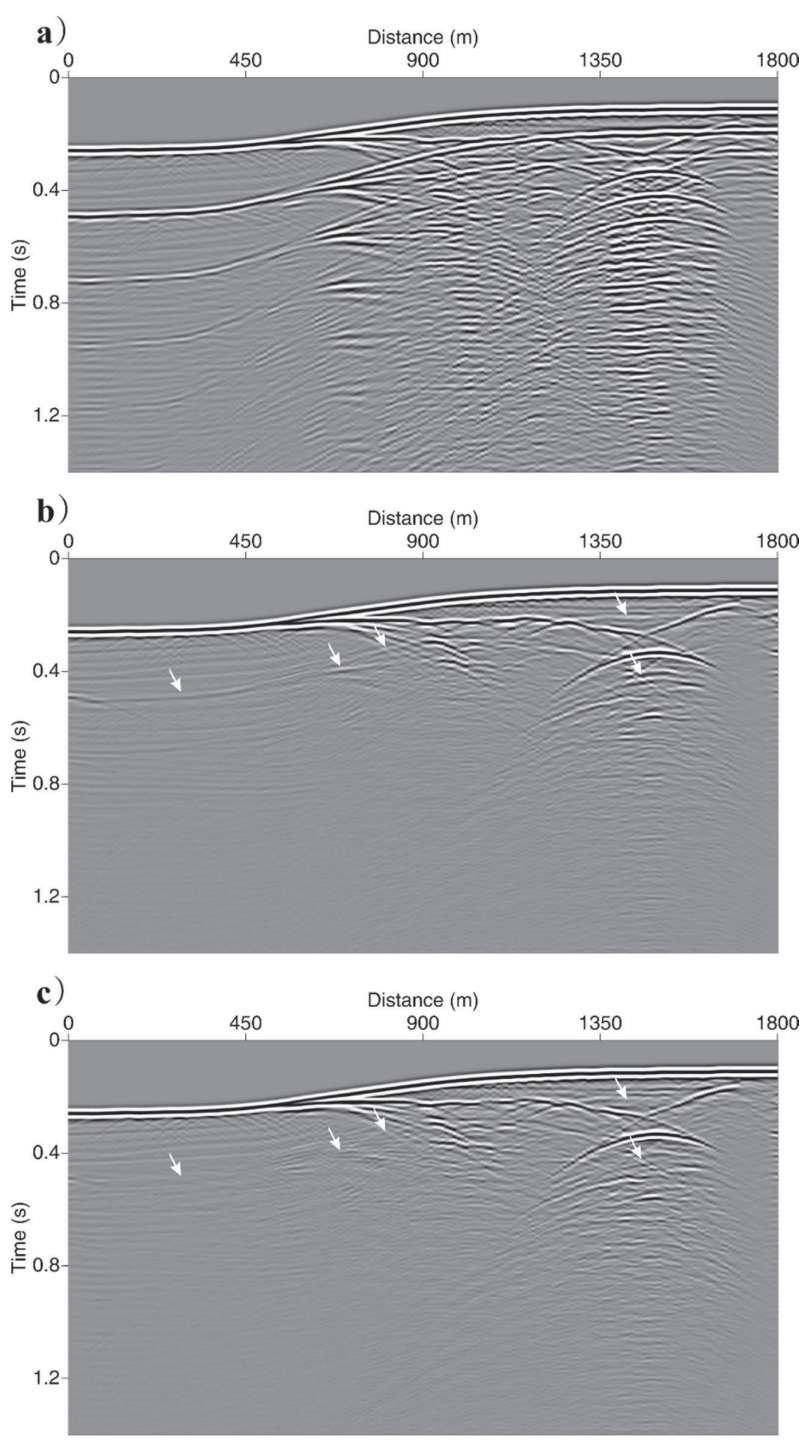

Figure 7: (a) The original zero-offset section; (b-c) the conservative primary estimation on zero-offset section resulted from EPSI after 60 iterations and our developed approach after 40 iterations.

approach after 40 iterations. It can be seen that both the approaches are effective for eliminating surface-related multiples. However, compared with the result of EPSI (Fig. 6b), the primaries resulted from our developed approach (Fig. 6c) contain less multiples residual, which is indicated by white arrows in the two figures. Figs. 6d and 6e show the final residual of EPSI and our developed approach respectively. The final residual can indicate the convergence of the algorithm. Despite 60 iterations, some residual is not explained with EPSI, especially at the shallow part of the shot-gather (indicated by arrows in Fig. 6d), which shows that a more desirable result needs more iterations. Whereas for 


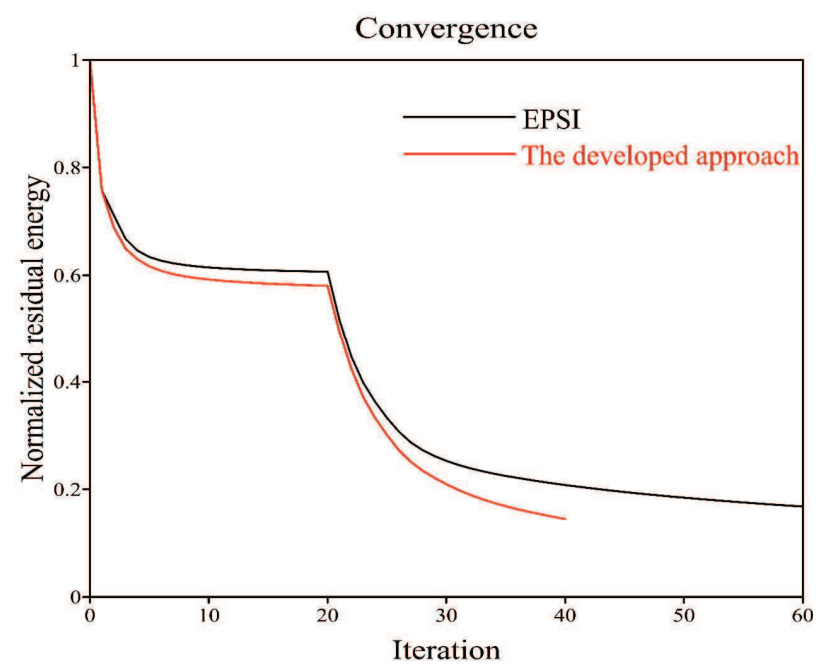

Figure 8: Convergence curves of EPSI and our developed approach.

our developed approach, the residual appears cleaner after only 40 iterations (Fig. 6e), which indicates a high convergence rate of our developed approach.

Applying the two approaches to all shots in the dataset, the appearance of the two approaches on zero-offset section can be shown in the Fig. 7, where the original zero-offset section, primaries estimated by EPSI, primaries estimated by our developed approach are sequentially displayed. Because of the strong water bottom, the water layer related multiples have a tremendous effect on the demultiple results. Such as in the result of EPSI (Fig. 7b), the first-order water-layer related multiples, which is between about $0.5 \mathrm{~s}$ at the left side and $0.2 \mathrm{~s}$ at the right side (indicated by white arrows), are not suppressed enough. Whereas in the result of our developed approach (Fig. 7c), the demultiple output appears cleaner.

The convergence curves of the two approaches are shown in Fig. 8, where the total residual energy is reduced to $16.8 \%$ for EPSI after 60 iterations, $14.4 \%$ for our developed approach after 40 iterations. The reason for the sharp decrease of the two curves at the 20th iteration is that the size of the picked window for imposing the sparsity is forced at the position of water-bottom primaries before the $20^{\text {th }}$ iteration, but relaxed after the $20^{\text {th }}$ iteration, so that the water-layer related multiples can be eliminated as much as possible during the first 20 iterations. The comparison for the two convergence curves shows our developed approach has a better convergence appearance than EPSI.

\subsection{A shallow-water field dataset}

As the final demonstration, we use a shallow-water field dataset to test the practicality of our developed approach. The dataset undergoes a series of preprocessing steps, including random noise removal, interpolation of missing shots and traces, deconvolution 

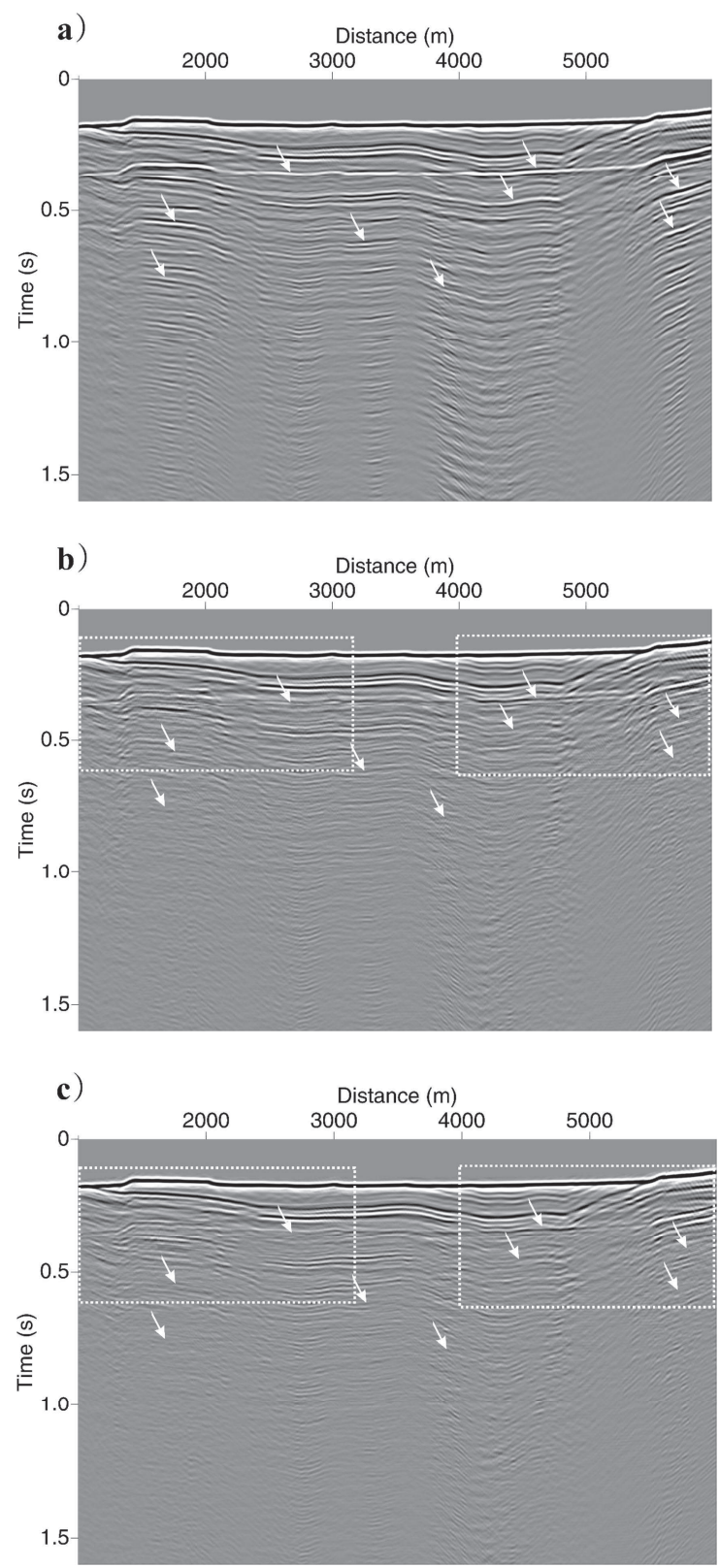

Figure 9: (a) The original zero-offset section of a shallow-water field dataset; (b-c) the conservative primary estimation on zero-offset section resulted from EPSI after 60 iterations and our developed approach after 60 iterations.

for the source bubble effects and so on. No deghosting is done and the direct wave is removed for all shots by muting. After preprocessing, the dataset includes 600 shots with each one containing 600 traces. All the sources and receivers are overlapped. 

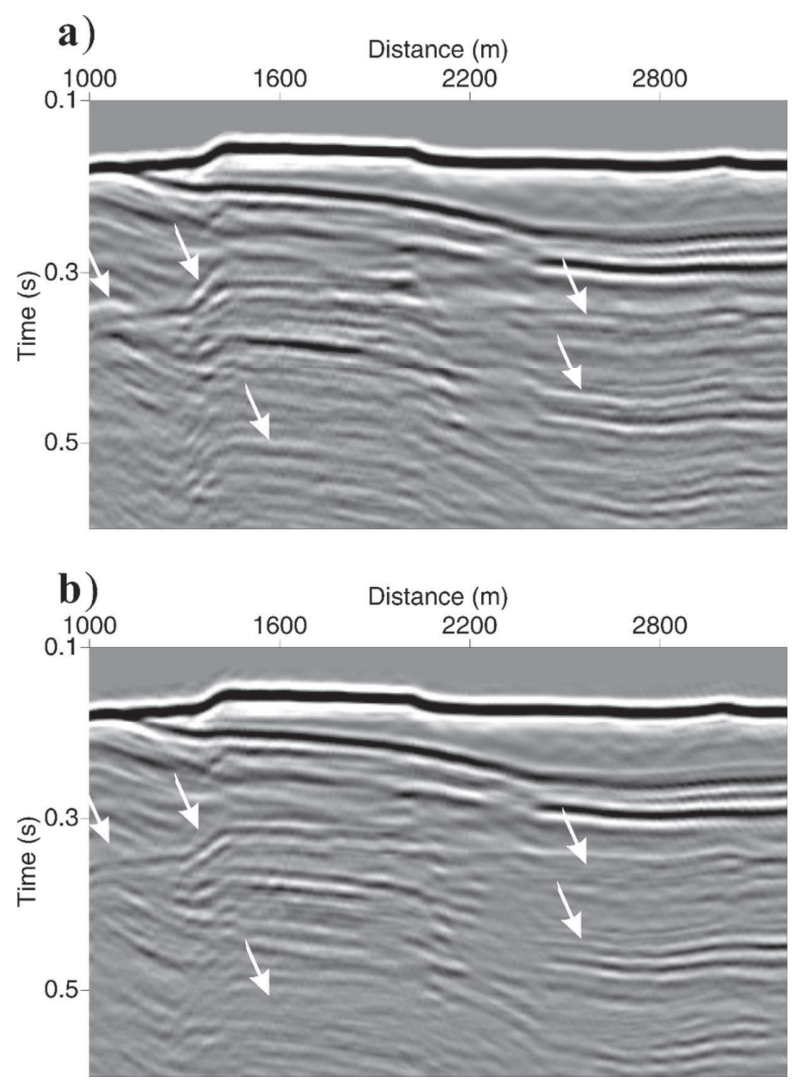

Figure 10: (a) The close-up view of the rectangular area at the left-side in Fig. 9b; (b) the close-up view for the rectangular area at the left-side in Fig. 9c. As indicated by arrows, the result in (b) contains less multiple residual than that in (a).

As with the previous two examples, the robustness of our developed approach is demonstrated by comparing it with EPSI. Both the approaches are taken 60 iterations to ensure the convergence. As a result, both the approaches are effective for attenuating multiples, as numerous multiple events in Fig. 9a are removed in Figs. 9b and 9c, which are indicated by arrows. However, compared with EPSI, our developed approach achieves a better behavior, as less multiple residual remained in Fig. 9c. This is further demonstrated in Fig. 10 and Fig. 11, which are the close-up views of the rectangular areas in Figs. 9b and 9c. This is mainly because that, our developed approach is not sensitive to amplitude inaccuracy of the field data as compared to EPSI.

The convergence curves of the two approaches are shown in Fig. 12. After 60 iterations, the total residual energy is reduced to $17.5 \%$ for EPSI and $11.9 \%$ for our developed approach. As with the second example, the reason for the sharp decrease of the two curves at the 20th iteration is that the size of the picked window for imposing the sparsity is forced at the position of water-bottom primaries before the $20^{\text {th }}$ iteration, but relaxed 

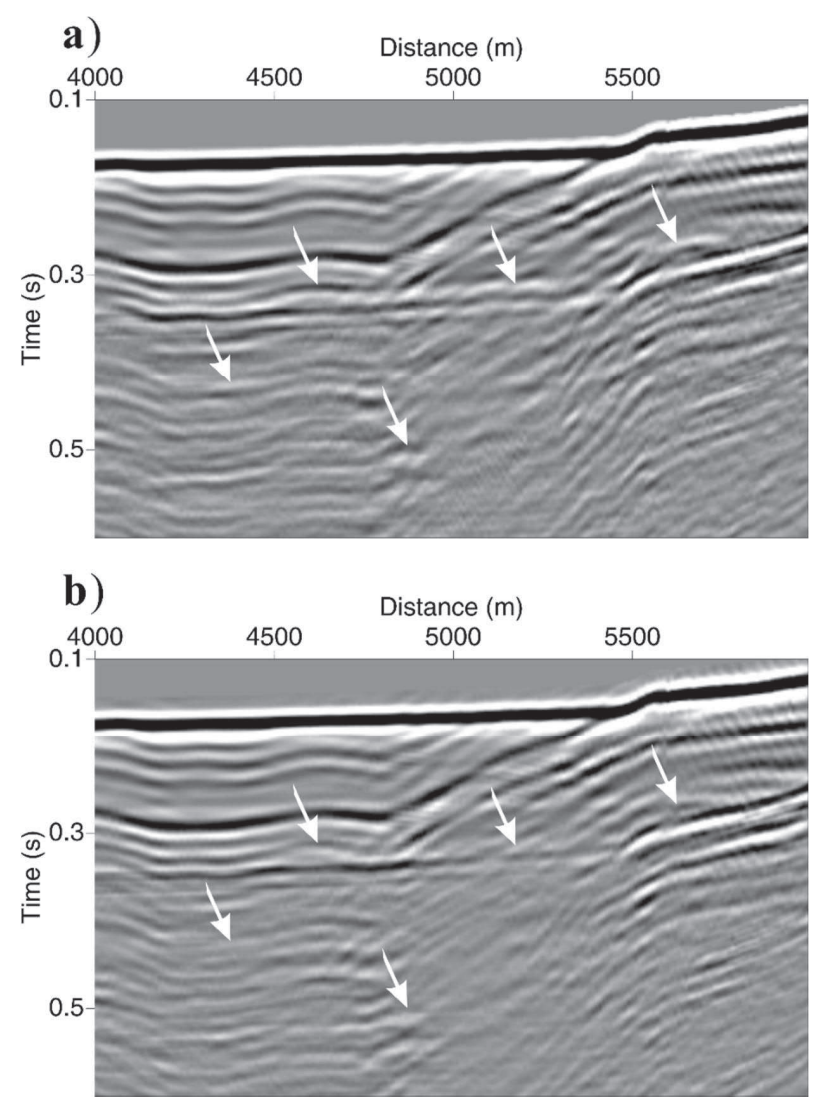

Figure 11: (a) The close-up view of the rectangular area at the right-side in Fig. 9b; (b) the close-up view for the rectangular area at the right-side in Fig. 9c. As indicated by arrows, the result in (b) contains less multiple residual than that in (a).

after the $20^{\text {th }}$ iteration, which contributes to efficiently explaining the water-layer related multiples. Overall, the example verifies the practicality of our developed approach.

\section{Conclusion and discussion}

We have developed a primary estimation approach based on the gradient-inversion framework. As with EPSI, the developed approach directly estimates the primaries by inversion which needs no subtraction process. But more importantly, the developed approach can yield a better primary estimation at less computational cost as compared to EPSI, which has been verified by two synthetic datasets and a shallow-water field dataset. The high efficiency of our developed approach lies in that a newly defined parameterization for primary-multiple model is adopted and an analytical step-length is derived for each update in the algorithm. The newly defined parameterization contributes to a better 


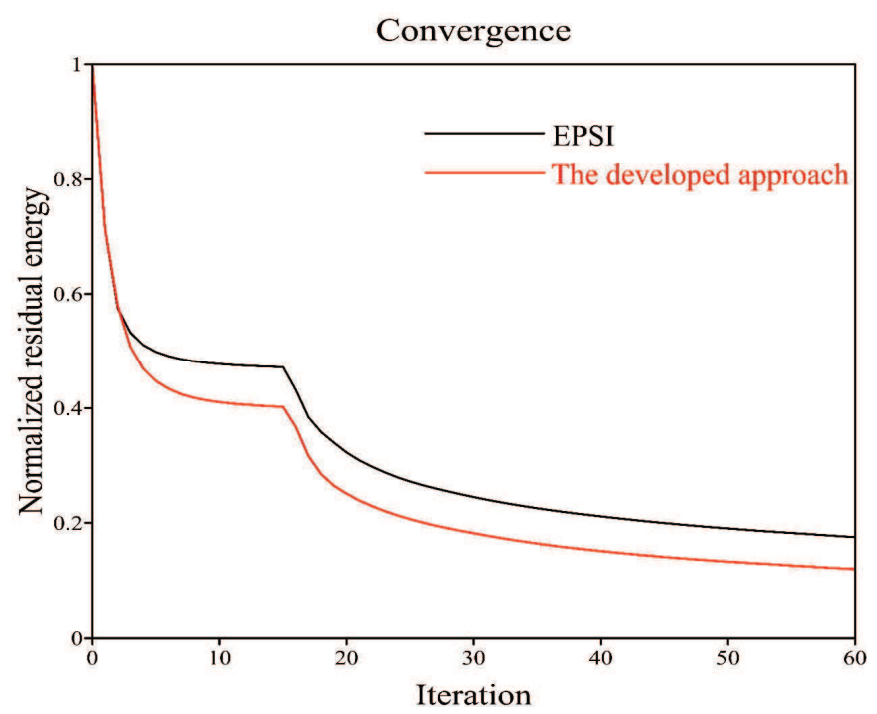

Figure 12: The convergence curves of EPSI and our developed approach.

convergence as compared to EPSI especially when the input data does not have a good amplitude consistency between primaries and multiples.

It should be noted that, there are still some problems in our developed approach that need to be further investigated: 1) The near-offset data reconstruction is not involved in the algorithm; 2) the developed approach cannot be suitable for the case that primaries and multiples are strongly interfered. Thus, we hope that in our future studies, these issues can be successfully tackled, so that our approach can have a broad application base.

\section{Acknowledgments}

We are especially grateful to Ru-Shan $\mathrm{Wu}$ for his support to our research group. The research is supported by National Natural Science Foundation of China (Grants 41730425 and 41430321) and National Oil and Gas Major Project of China (Grant 2017ZX05008-007).

\section{Appendix A: Derivation of analytical step-lengths for our developed approach}

Ignoring the regularization term, the objective function in Eq. (2.8) can be rewritten as

$$
J=\sum_{i, j} \sum_{w}\left|\mathbf{P}-\mathbf{X}_{0, i}^{P_{0}} S_{i}-\mathbf{X}_{0, i}^{M} \mathbf{P}\right|^{2}
$$


For alternately inverting for the two types of primary impulse response, the gradients of $\mathbf{X}_{0, i}^{P_{0}}$ and $\mathbf{X}_{0, i}^{M}$ can be calculated with:

$$
\left\{\begin{array}{l}
\Delta \mathbf{X}_{0, i}^{M}=-\frac{\partial J}{\partial \mathbf{X}_{0, i}^{M}}=-\left(\mathbf{P}-\mathbf{X}_{0, i}^{P_{0}} S_{i}-\mathbf{X}_{0, i}^{M} \mathbf{P}\right) \mathbf{P}^{H} \\
\Delta \mathbf{X}_{0, i}^{P_{0}}=-\frac{\partial J}{\partial \mathbf{X}_{0, i}^{P_{0}}}=-\left(P-\mathbf{X}_{0, i}^{P_{0}} S_{i}-\mathbf{X}_{0, i}^{M} \mathbf{P}\right) S_{i}^{H}
\end{array}\right.
$$

After imposing the sparsity to $\Delta \mathbf{X}_{0, i}^{M}$ and $\Delta \mathbf{X}_{0, i}^{P_{0}}, \mathbf{X}_{0, i+1}^{P_{0}}$ and $\mathbf{X}_{0, i+1}^{M}$ can be expressed as

$$
\left\{\begin{array}{l}
\mathbf{X}_{0, i+1}^{M}=\mathbf{X}_{0, i}^{M}+\alpha_{1} \Delta \mathbf{X}_{0, i^{\prime}}^{M} \\
\mathbf{X}_{0, i+1}^{P_{0}}=\mathbf{X}_{0, i}^{P_{0}}+\alpha_{2} \Delta \mathbf{X}_{0, i^{\prime}}^{P_{0}}
\end{array}\right.
$$

where $\alpha_{1}$ and $\alpha_{2}$ are the step-length, which are to be obtained. Substitute Eq. (A.3) into Eq. (A.1), the residual energy in the $i+1$ iteration can be shown as:

$$
J_{i+1}=\sum_{j, k} \sum_{w}\left|\mathbf{P}-\left(\mathbf{X}_{0, i}^{P_{0}}+\alpha_{2} \Delta \mathbf{X}_{0, i}^{P_{0}}\right) S_{i}-\left(\mathbf{X}_{0, i}^{M}+\alpha_{1} \Delta \mathbf{X}_{0, i}^{M}\right) \mathbf{P}\right|^{2} .
$$

In Eq. (A.4), the optimal values for $\alpha_{1}$ and $\alpha_{2}$ should minimize the residual energy. Therefore, the problem for calculating $\alpha_{1}$ and $\alpha_{2}$ in Eq. (A.4) is equivalent to a minimum problem for a 2-variables function.

For simplicity, we rewrite Eq. (A.4) as

$$
J\left(\alpha_{1}, \alpha_{2}\right)=\sum_{i, j, k}\left|\mathbf{W}_{0}-\alpha_{1} \mathbf{W}_{1}-\alpha_{2} \mathbf{W}_{2}\right|^{2},
$$

where $\mathbf{W}_{0}=\mathbf{P}-\mathbf{X}_{0, i}^{P_{0}} S_{i}-\mathbf{X}_{0, i}^{M} \mathbf{P}, \mathbf{W}_{1}=\Delta \mathbf{X}_{0, i}^{M} \mathbf{P}$ and $\mathbf{W}_{2}=\Delta \mathbf{X}_{0, i}^{P_{0}} S_{i}$. As $J$ is a convex function, the extreme value can be obtained when the partial derivatives are zeros:

$$
\left\{\begin{array}{l}
\frac{\partial J}{\partial \alpha_{1}}=\alpha_{1} \sum_{i, j, k} \mathbf{W}_{1, i j k}^{2}+\alpha_{2} \sum_{i, j, k} \mathbf{W}_{1, i j k} \mathbf{W}_{2, i j k}-\sum_{i, j, k} \mathbf{W}_{0, i j k} \mathbf{W}_{1, i j k}=0, \\
\frac{\partial J}{\partial \alpha_{2}}=\alpha_{1} \sum_{i, j, k} \mathbf{W}_{1, i j k} \mathbf{W}_{2, i j k}+\alpha_{2} \sum_{i, j, k} \mathbf{W}_{2, i j k}^{2}-\sum_{i, j, k} \mathbf{W}_{0, i j k} \mathbf{W}_{2, i j k}=0 .
\end{array}\right.
$$

Solve Eq. (A.6), $\alpha_{1}$ and $\alpha_{2}$ can be simultaneously obtained:

$$
\left\{\begin{array}{l}
\alpha_{1}=\frac{\mathbf{B}_{2} \mathbf{A}_{2}-\mathbf{B}_{1} \mathbf{A}_{3}}{\mathbf{A}_{2}^{2}-\mathbf{A}_{1} \mathbf{A}_{3}} \\
\alpha_{2}=\frac{\mathbf{B}_{1} \mathbf{A}_{2}-\mathbf{B}_{2} \mathbf{A}_{1}}{\mathbf{A}_{2}^{2}-\mathbf{A}_{1} \mathbf{A}_{3}}
\end{array}\right.
$$

where $\mathbf{A}_{1}=\sum_{i, j, k} \mathbf{W}_{1, i j k}^{2}, \mathbf{A}_{2}=\sum_{i, j, k} \mathbf{W}_{1, i j k} \mathbf{W}_{2, i j k}, \mathbf{A}_{3}=\sum_{i, j, k} \mathbf{W}_{2, i j k}^{2}, \mathbf{B}_{1}=\sum_{i, j, k} \mathbf{W}_{0, i j k} \mathbf{W}_{1, i j k}$ and $\mathbf{B}_{2}=\sum_{i, j, k} \mathbf{W}_{0, i j k} \mathbf{W}_{2, i j k}$. 


\section{References}

[1] R. H. Baardman, D. J. Verschuur, R. G. van Borselen, M. O. Frijlink, and R. F. Hegge. Estimation of primaries by sparse inversion using dual sensor data. in 80th Annual International Meeting, SEG, Expanded Abstracts, 3468-3472, 2010.

[2] L. Bai, H. Lu, Y. Liu and M. Khan. A fast joint seismic data reconstruction by sparsitypromoting inversion. Geophys. Prospect., 65: 926-940, 2017.

[3] A. J. Berkhout. Seismic migration, imaging of acoustic energy by wave field extrapolation. A, Theoretical aspects 2nd ed, Elsevier, 1982.

[4] A. J. Berkhout and D. J. Verschuur. Estimation of multiple scattering by iterative inversion, part I: Theoretical considerations. Geophysics, 62: 1586-1595, 1997.

[5] J. Biersteker. MAGIC: Shell's surface multiple attenuation technique. in 71st Annual International Meeting, SEG, Expanded Abstracts, 1301-1304, 2001.

[6] X. Hu, J. Qin, J. Liu and J. Hembd. Estimation of Primaries by Sparse Inversion in Shallow Water - Practical Challenges and Strategies. in 77th Annual International Meeting, EAGE, Extended Abstracts, 270-284, 2015.

[7] B. Hung, K. L. Yang, J. Zhou, and Q. L. Xia. Shallow Water Demultiple. in ASEG, Expanded Abstracts, 1-4, 2010.

[8] B. Jumah, and F. J. Herrmann. Dimensionality-reduced estimation of primaries by sparse inversion. Geophys. Prospect., 62: 972-993, 2014.

[9] T. T. Y. Lin and F. J. Herrmann. Unified compressive sensing framework for simultaneous acquisition with primary estimation. in 79th Annual International Meeting, SEG, Expanded Abstracts, 3113-3117, 2009.

[10] T. T. Y. Lin and F. J. Herrmann. Robust estimation of primaries by sparse inversion via onenorm minimization. Geophysics, 78(3): R133-R150, 2013.

[11] T. T. Y. Lin and F.J. Herrmann. Estimation of primaries by sparse inversion with scatteringbased multiple predictions for data with large gaps. Geophysics, 81(3): V183-V197, 2016.

[12] Y. Liu, D. Jin, X. Chang, P. Li, H. Sun, and Y. Luo. Multiple subtraction using statistically estimated inverse wavelets. Geophysics, 75(6): WB247-WB254, 2010.

[13] G. A. Lopez and D. J. Verschuur. SRME and estimation of primaries by sparse inversion: a hybrid approach. in 82th Annual International Meeting, SEG, Expanded Abstracts, 1-5, 2012.

[14] G. A. Lopez and D. J. Verschuur. Closed-loop surface-related multiple elimination and its application to simultaneous data reconstruction. Geophysics, 80(6): V189-V199, 2015.

[15] A. G. Nekut and D. J. Verschuur. Minimum energy adaptive subtraction in surface-related multiple attenuation. in 68th Annual International Meeting, SEG, Expanded Abstracts, 15071510, 1998.

[16] J. Paffenholz, B. Mclain, J. Zaske and P. J. Keliher. Subsalt multiple attenuation and imaging observations from the Sigsbee2B synthetic data set. in 72nd SEG Annual International Meeting (Expanded Abstract), pp. 2122-2125, 2002.

[17] T. Savels, K. de Vos and J. W. de Maag. Surface multiple attenuation through sparse inversion; results for complex synthetics and real data. First Break, 29: 55-64, 2011.

[18] G. Toxopeus, R. G. van Borselen, R. H. Baardman, L. Auer, and E. Odegaard. Advanced geohazards assessment in shallow water through the estimation of primaries by sparse inversion. in 81st Annual International Meeting, SEG, Expanded Abstracts, 3526-3530, 2011.

[19] G. J. A. van Groenestijn and D. J. Verschuur. Estimating primaries by sparse inversion and application to near-offset data reconstruction. Geophysics, 74(3): A23-A28, 2009.

[20] G. J. A. van Groenestijn and D. J. Verschuur. Estimation of primaries and near-offset recon- 
struction by sparse inversion, Marine data applications. Geophysics, 74(6): R119-R128, 2009.

[21] G. J. A. van Groenestijn and D. J. Verschuur. Estimation of primaries by sparse inversion applied to up/down wavefields. in 79th Annual International Meeting, SEG, Expanded Abstracts, 3143-3147, 2009.

[22] G. J. A. van Groenestijn and D. J. Verschuur. Estimation of primaries by sparse inversion from passive seismic data. Geophysics, 75(4): SA61-SA69, 2010.

[23] G. J. A. van Groenestijn and D. J. Verschuur. Using surface multiples to estimate primaries by sparse inversion from blended data. Geophys. Prospect., 59: 10-23, 2011.

[24] G. J. A. van Groenestijn and W. Ross. Primary estimation on OBC data by sparse inversion. in 81st Annual International Meeting, SEG, Expanded Abstracts, 3531-3535, 2011.

[25] J. W. Vrolijk, E. Verschuur, and G. Lopez. Integrated receiver deghosting and closed-loop surface-multiple elimination. Geophysics, 82(4): T133-T141, 2017.

[26] D. J. Verschuur, A. J. Berkhout, and C. P. A. Wapenaar. Adaptive surface-related multiple elimination. Geophysics, 57: 1166-1177, 1992.

[27] P. Wang, H. Jin, S. Xu, and Y. Zhang. Model-based water-layer demultiplex. in 81st Annual International Meeting, SEG, Expanded Abstracts, 3551-3555, 2011.

[28] Y. Wang. Multiple prediction through inversion: A fully data-driven concept for surfacerelated multiple attenuation. Geophysics, 69: 547-553, 2004.

[29] S. Yuan, S. Wang, F. Yuan and Y. Liu. The influence of errors in the source wavelet on inversion-based surface-related multiple attenuation. Geophys. Prospect., in press, 2017.

[30] Q. Fu, Y. Luo, P. G. Kelamis, S. Huo, G. Sindi, S. Hsu and A. B. Weglein. The inverse scattering series approach towards the elimination of land internal multiples. in 80st Annual International Meeting, SEG, Expanded Abstracts, 3456-3461, 2010.

[31] Q. Fu and A. B. Weglein. Internal multiple attenuation on Encana data. in 84st Annual International Meeting, SEG, Expanded Abstracts, 4118-4123, 2014.

[32] D. Zhang and G. T. Schuster. Least-squares reverse time migration of multiples. Geophysics, 79(1), S11-S21, 2014.

[33] D. Zhang, T. W. Fei and Y. Luo. Improving reverse time migration angle gathers by efficient wavefield separation. Geophysics, 83(2), S187-S195, 2018.

[34] A. Osen, L. Amundsen and A. Reitan. Removal of water-layer multiples from multicomponent sea-bottom data: Geophysics, 64, 838-851, 1999.

[35] A. Osen, L. Amundsen and A. Reitan. Toward optimal spatial filters for demultiple and wavefield splitting of ocean-bottom seismic data: Geophysics, 67, 1983-1990, 2002.

[36] Y. Liu, X. Chang, D. Jin, R. He, H. Sun, and Y. Zheng. Reverse time migration of multiples for subsalt imaging. Geophysics, 76(5), WB209-WB216, 2011.

[37] X. Liu, Y. Liu, H. Hu, P. Li, and M. Khan. Imaging of first-order surface-related multiples by reverse-time migration. Geophys. J. Int., 208, 1077-1087, 2017. 